\title{
Covid-19: We have good treatments for omicron, but questions remain, say doctors
}

\section{Emma Wilkinson freelance journalist}

Although the impact of omicron has largely been mitigated by vaccination, prior SARS-CoV-2 infection, and better treatments, general medical wards still face high numbers of patients, with many parts of the UK still bracing themselves for the peak, say clinicians.

The most recent report from the Intensive Care National Audit and Research Centre (ICNARC), ${ }^{1}$ with data up to 7 January, showed that 629 had been patients admitted to critical care with covid-19 in the previous fortnight. As a proportion of overall hospital admissions, the figures for covid related critical care are at the lowest they have been since reporting began in April 2020.

On 10 January 18655 patients were in hospital with covid-19, with daily admissions reaching $2332 .{ }^{2}$ Simon Ashworth, a consultant in intensive care medicine at Imperial College Healthcare NHS Trust, told The BMJ, "Hospitals are very full, emergency departments are really busy, there are lots of admissions, difficulties in getting people into care homes, and we need to isolate covid patients from others.”

Ashworth said the situation in intensive care wasn't comparable with that in January 2021 but remained challenging, with a fluctuating acuity of patients. "We still have expansion beds open well beyond our normal capacity because we have a lot of other urgent and emergency patients too. A higher than normal proportion of patients are on ventilators, and beds to discharge patients into are hard to come by."

He remains cautious about what is ahead, given that every surge has not "been quite as expected."

\section{Clearer picture on treatments}

While staff shortages are a major problem across all teams at the moment, large randomised controlled trials have provided clinicians with some guidance on treatments.

"We are in a different place," said Lisa Spencer, a respiratory consultant and honorary secretary of the British Thoracic Society. "Patients who are admitted get dexamethasone and tocilizumab if they meet certain criteria.” Remdesivir was used quite a lot to treat hospital patients in earlier waves but much less so now, she added.

Respiratory support units have been set up in many large hospitals to provide a higher level of monitoring and respiratory intervention than would be expected in a routine ward environment. "Respiratory support units will be part of the reason why ICUs are not filling up. We know that giving CPAP [continuous positive airway pressure] is likely to stop the patient getting to intensive care," said Spencer.
Beverley Hunt, a consultant in thrombosis and haemostasis at Kings Healthcare Partners, an academic health science centre in London, said large studies had also removed the uncertainty around thromboprophylaxis. "We have had multiple international trials which all show that if you have moderate covid, by which I mean you need low flow oxygen, but you don't need mechanical ventilation or CPAP, you have a better clinical outcome with therapeutic low molecular weight heparin, but those with severe covid need to have standard doses of thromboprophylactic."

She added, "My impression so far is that we're not seeing such severe disease and therefore we're not seeing such high rates of thrombosis overall."

Monoclonal antibodies are a potential option for some patients. But while Ronapreve, the combination of casirivimab and imdevimab, had been available in hospitals for some weeks, it was found to have significantly decreased efficacy against omicron.

The Recovery trial is preparing to add the neutralising monoclonal antibody sotrovimab and the antiviral molnupiravir to its platform of treatments for hospital patients. By chance, the team led by Anthony Kerry, consultant respiratory physician at the Great Western Hospital in Swindon, recruited the 46 oooth participant to Recovery at the end of 2021.

“Apart from Ronapreve a lot of treatment protocols are the same for omicron, but the landscape is changing because more people are coming in with milder illness. We haven't got all the answers and therapies yet, and so we do need more research," Kerry said.

\section{Proactive prevention}

One big change in December was the introduction of covid medicine delivery units (CMDUs) to offer either sotrovimab or molnupiravir to eligible patients with mild disease in the community with the aim of preventing hospital admission. ${ }^{3}$ Anna Goodman, a consultant in infectious disease and general medicine at Guy's and St Thomas' NHS Foundation Trust, said that more than 2000 patients had been referred to its unit since mid-December. "The most effective covid preventive treatment is vaccination," Goodman said. "These are treatments for patients who have been vaccinated but are not able to respond to vaccines so are vulnerable.” People who are eligible, who include those who have recently had chemotherapy or who cannot generate antibodies because of a medical condition, must have had a confirmatory PCR and onset of covid symptoms within the past five days.

"We look forward to understanding more about how people who have been vaccinated may perhaps 
benefit from these treatments," Goodman said, adding, "Every CMDU has seen very large numbers of patients, and we have been open every single day except Christmas Day and New Year's Day.”

Thomas Jackson, a visiting consultant in geriatric medicine at University Hospitals Birmingham NHS Foundation Trust, who described hospitals as "just about coping," said a significant number of older patients with covid had always presented without the classic symptoms. "The feeling is that now with omicron there are more atypical presentations in general, and that's amplified in the older population," he said.

The difficulty in this population was that there are no clear guidelines yet on the treatments that would make a difference, he explained. "The way that dexamethasone and IL6 inhibitors are given is around respiratory failure and oxygen requirements. What we don't yet know is whether these treatments would be of any benefit [to people] with atypical symptoms," he said.

1 Intensive Care National Audit and Research Centre. Covid-19 report. Jan 2022. https://www.icnarc.org/Our-Audit/Audits/Cmp/Reports.

2 Healthcare in the UK: coronavirus (covid-19) in the UK. Gov.UK. Jan 2022. https://coronavirus. data.gov.uk/details/healthcare.

3 Department of Health and Social Care. Interim clinical commissioning policy: neutralising monoclonal antibodies or antivirals for non-hospitalised patients with COVID-19. Dec 2021. https://www.england.nhs.uk/coronavirus/wp-content/uploads/sites/52/2021/12/C1530_Interimclinical-commissioning-policy-neutralising-monoclonal-antibodies-or-antivirals-for-non-hospitali.pdf.

This article is made freely available for personal use in accordance with BMJ's website terms and conditions for the duration of the covid-19 pandemic or until otherwise determined by BMJ. You may download and print the article for any lawful, non-commercial purpose (including text and data mining) provided that all copyright notices and trade marks are retained. 